\title{
Lésions cérébrales démyélinisantes découvertes chez un patient immunocompétent VIH-1+
}

\section{Demyelinating cerebral lesions in an immunocompetent HIV-1 patient}

\author{
D. Nkoghe*, M. Moutschen, J. Demonty \\ Service des maladies infectieuses et de médecine interne générale, centre hospitalier universitaire de Liège, Liège, Belgique \\ Reçu le 11 décembre 2007 ; accepté le 19 juin 2008 \\ Disponible sur Internet le 29 août 2008
}

\begin{abstract}
Résumé
Des lésions cérébrales démyélinisantes similaires à la sclérose en plaques ont été découvertes chez un patient de 32 ans VIH immunocompétent. À l'histologie, une inflammation avec nécrose, démyélinisation et destruction des axones a été notée. Divers tests sérologiques, des cultures spécifiques et la PCR pour plusieurs agents infectieux étaient négatifs. La corticothérapie et les antibiotiques se sont révélés sans effet. Une trithérapie antirétrovirale et l'ablation d'une des lésions ont permis la résorption complète des nodules, sans récurrence. Cette atteinte démyélinisante semblait être la première manifestation d'une primo-infection. L'implication du VIH dans la genèse du processus et sa pérennisation a été suspectée mais le mécanisme responsable n'est pas clair, probablement auto-immun. L'inhibition de la réplication virale induite par le traitement combiné réduirait les effets dysimmunitaires du VIH. Notre présentation constitue la première description de l'efficacité indirecte d'une trithérapie sur des lésions cérébrales démyélinisantes chez un patient immunocompétent.
\end{abstract}

(C) 2008 Elsevier Masson SAS. Tous droits réservés.

\begin{abstract}
We report the case of a 32-year-old immunocompetent HIV patient, presenting with acute demyelinating leukoencephalopathy. The patient displayed clinical and radiological features similar to multiple sclerosis. Histology revealed inflammation with necrosis, demyelination and destruction of axons. Serum tests were negative for various infectious agents as well as specific cultures and PCR. Corticotherapy and many antibiotic treatments failed. Resorption of the lesions occurred after partial excision and highly-active antiretroviral therapy (HAART). No recurrence was noted. This demyelinating cerebral disease was considered as the primary manifestation of HIV infection. HIV implication in the genesis of the process and its perpetuating this condition was suspected, but the mechanism is unclear. Dysimmune consequences related to the early course of HIV infection could be prevented by antiretroviral treatment. This study was the first description of tritherapy effectiveness on HIV related multiple sclerosis (MS)-like illness.
\end{abstract}

(C) 2008 Elsevier Masson SAS. Tous droits réservés.

Mots clés : Leucoencéphalopathie ; VIH

Keywords: HIV; Leukoencephalopathy

\section{Introduction}

Le système nerveux central (SNC) est une cible privilégiée pour le VIH, qui y induit des manifestations neurologiques survenant à tous les stades de l'infection par deux mécanismes: atteinte directe par le VIH lui-même et infections

\footnotetext{
* Auteur correspondant. BP 5879, Libreville, Gabon.

Adresse e-mail : dnkoghe@ hotmail.com (D. Nkoghe).
}

et tumeurs opportunistes du fait de l'immunodépression. Ces manifestations peuvent globalement se subdiviser en quatre grands groupes de syndromes cliniques distincts: les syndromes focaux, méningés, démentiels et médullaires. L'apport de l'imagerie (notamment l'imagerie par résonance magnétique [IRM]) reste déterminant pour le diagnostic de nombreuses affections cérébrales. Ainsi, au niveau de l'IRM, ces atteintes se répartissent en lésions focales ou diffuses, circonscrites ou non, uniques ou multiples, avec ou sans effet de masse. La confrontation entre le mode d'installation des troubles neurologiques 
(aigu, subaigu ou progressif) et l'aspect des lésions est déterminant pour établir le diagnostic. Les données de la ponction lombaire, les tests biologiques spécifiques et, dans certains cas, l'histologie peuvent apporter des éléments de confirmation. Au plan thérapeutique, bien que certaines de ces pathologies disposent de traitement spécifique aux bénéfices variables, pour bien d'autres, il n'en existe pas.

Les infections constituent les causes majoritaires des atteintes du SNC, avec en première place la toxoplasmose. Cette affection parasitaire, habituellement asymptomatique chez l'homme, va se manifester, au stade de l'immunodéficience sévère, sous la forme d'abcès cérébraux donnant un syndrome tumoral ou focal accompagné de fièvre et de signes d'hypertension intracrânienne. Le lymphome cérébral primitif et la leucoencéphalopathie multifocale progressive (LEMP) donnent aussi des déficits focaux et surviennent quasiment au même stade évolutif de l'infection VIH. Cependant, ils comportent des différences scannographiques et histologiques caractéristiques. Ainsi, le lymphome primitif va induire une atteinte mono- ou bifocale des noyaux gris centraux ou lobaires, au niveau supratentoriel avec un effet de masse peu important et une prise de contraste nodulaire à bords flous ; alors que pour la LEMP, c'est essentiellement la substance blanche sous-corticale qui est touchée de façon multifocale au niveau du carrefour ventriculaire, et il n'y a ni effet de masse ni prise de contraste. De plus, l'histologie les différencie par la mise en évidence, d'une part, d'une infiltration lymphocytaire tumorale périvasculaire infiltrante et d'autre part, des lésions démyélinisantes.

Outre ces pathologies liées à l'immunodépression, l'atteinte directe SNC par le VIH, suite à son neurotropisme, va se traduire par des différents types de lésions (myélopathie vacuolaire, méningite aseptique, encéphalite) observées à divers stades de l'infection VIH. L'encéphalite VIH semble être la plus fréquente et est corrélée à l'importance du déficit immunitaire. Elle se manifeste sous forme de syndrome dépressif, de troubles de la mémoire, un ralentissement idéomoteur, des troubles de la concentration et de l'attention, le tout évoluant vers un syndrome démentiel de type sous-cortical. L'IRM révèle une atrophie cérébrale et une atteinte diffuse multifocale sous-corticale de la substance blanche.

Les lésions démyélinisantes sont une cause rare d'atteinte du SNC, et la LEMP demeure la principale. Cette affection est caractérisée par une destruction de la substance blanche par infection puis destruction des oligodendrocytes par un papovavirus humain, le virus JC. Elle survient chez $4 \%$ des sidéens. En dehors du VIH, de nombreuses affections démyélinisantes de la substance blanche sont décrites: les maladies métaboliques congénitales (phénylcétomurie, maladie de Gaucher, maladie de Niemann-Pick, etc.) et les maladies démyélinisantes primitives (l'encéphalomyélite postinfectieuse, la sclérose en plaques [SEP], etc.). La SEP est la plus importante de ces maladies et est caractérisée par des plaques disséminée de démyélinisation dans le cerveau et la moelle épinière, responsables des signes neurologiques très polymorphes, évoluant par phases successives de poussées et de rémissions. Son étiologie reste à ce jour inconnue; on note toutefois la conjonction des facteurs génétiques, environnementaux, immuns et infectieux dans la genèse de la maladie. Récemment, il a été rapporté l'existence intrigante de lésions démyélinisantes mimant la SEP chez des patients à différents stades de l'infection VIH [1-5]. Bien qu'aucune particule virale n'ait été détectée, le rôle du VIH est suspecté. On peut aussi se demander s'il s'agit là de deux entités différentes.

Dans ce cadre, nous présentons les données d'un patient de la trentaine chez qui des lésions démyélinisantes cérébrales ont été découvertes en même temps que sa séropositivité pour le VIH, et dont l'évolution a été favorable sous traitement antirétroviral combiné.

\section{Description clinique du cas}

Un patient caucasien âgé de 32 ans dont la sérologie VIH s'était révélée négative six mois auparavant est admis pour perte brutale de l'acuité visuelle de l'œil droit. L'examen ophtalmologique met en évidence un scotome central et il n'y a aucun signe inflammatoire au fond d'œil. Le scanner cérébral réalisé par la suite est normal. L'étude biochimique, cytobactériologique et histologique du liquide céphalorachidien ne montre pas d'anomalie. Une névrite optique rétrobulbaire est donc évoquée et un traitement par prednisolone en intraveineux instauré. La symptomatologie régresse en une semaine. Des potentiels évoqués et une IRM cérébrale sont programmés en ambulatoire, mais le patient ne se présente pas.

Trois mois plus tard, le patient est réadmis dans un tableau de syndrome frontal, troubles de la mémoire et asymétrie des réflexes à droite. L'électroencéphalogramme montre des rythmes lents dans l'hémisphère gauche. À l'IRM, trois nodules de 6,5 et $3 \mathrm{~cm}$ de diamètre sont mis en évidence au niveau de la substance blanche, respectivement, en frontal, pariétal et temporal (Fig. 1). Ces nodules apparaissent hypo-intenses en T1 et hyperintenses après injection de gadolinum ainsi qu'en T2, surtout dans la partie centrale. La lésion frontale traduit un effet de masse important.

La ponction lombaire n'est pas réalisée. La présence de ces trois lésions conduit à la réalisation d'une biopsie stéréotaxique. À l'histologie, il n'y a pas d'arguments en faveur d'une tumeur néoplasique ; cependant, la présence de lésions de nécrose permet de suspecter une origine infectieuse. Des tests sanguins sur les fragments biopsiés sont ainsi effectués : les cultures standard et sur milieux spécifiques (y compris pour les mycobactéries) sont stériles; la recherche par PCR de Toxoplasma gondii, Treponema pallidum, Borrelia borgdurfery, des virus des hépatites (A, B et C), HSV, HHV-6, VZV, virus des oreillons, CMV, virus d'Epstein-Barr et le virus JC est négative. Les anticorps antiVIH sont détectés et leur spécificité confirmée par le western blot. Le dosage des lymphocytes CD4 se situe à 687 par millimètre cube et la charge virale est quantifiée à 144000 copies par millilitre. Malgré l'absence d'agents infectieux identifiés, un traitement à visée anti-infectieuse est instauré. Elle associe la ceftriaxone, l'amphotéricine B, la sulfadiazine-pyriméthamine et de fortes doses de prednisolone, pendant quatre semaines, sans succès. Aussi, après trois mois d'hospitalisation sans bénéfice notable, une craniotomie est préconisée, conduisant à une exérèse partielle de la lésion frontale seule. Les suites opératoires sont simples. L'IRM postopératoire confirme les données 


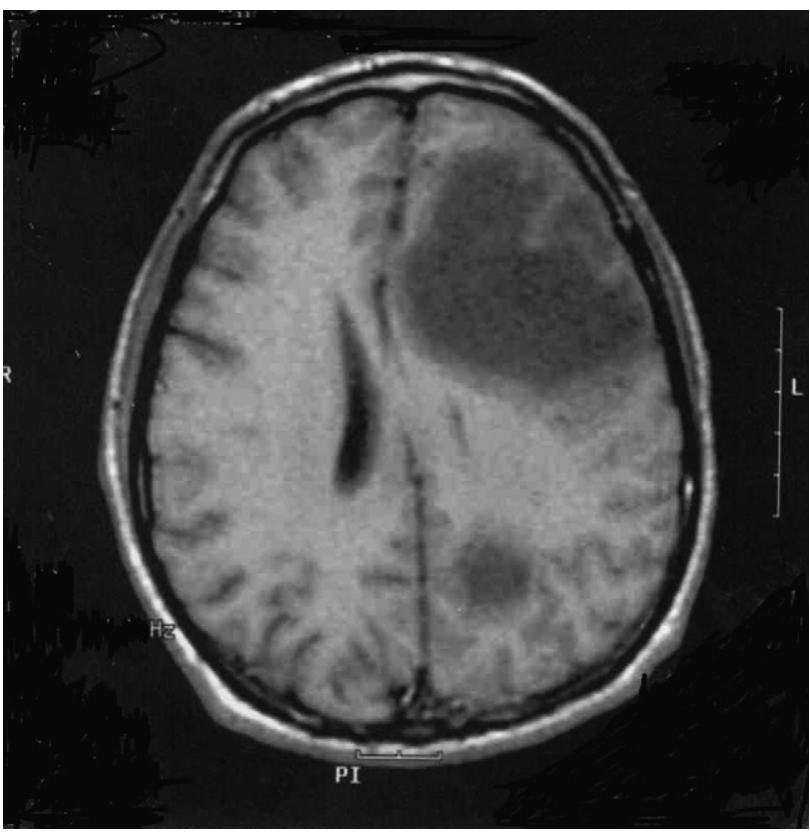

Fig. 1. IRM du cerveau lors du diagnostic, volumineux nodule frontal avec prise de contraste et signe de compression ; nodule temporal non compressif.

Magnetic resonance imaging of the brain during the diagnosis: voluminous frontal lesion taking contrast and sign of compression; no compression with the temporal lesion.

de l'intervention par la diminution importante du diamètre de cette lésion. L'analyse histologique de la pièce d'exérèse met en évidence des stigmates d'inflammation avec nécrose, démyélinisation, mais aussi destruction des axones. Il n'est pas noté de signe de prolifération astrocytaire. Une fois de plus, aucun agent infectieux n'est mis en évidence par la culture et la PCR, y compris les antigènes du VIH et du virus JC. L'ensemble de ces éléments plaide néanmoins en faveur d'une atteinte démyélinisante de la substance grise. Tenant compte du niveau de la charge virale et de l'état clinique du patient, un traitement antirétroviral combiné est initié dans les suites de l'intervention. Une régression rapide et spectaculaire des symptômes neurologiques est constatée en deux semaines et la sortie du patient est autorisée. Un contrôle IRM à trois mois confirme les données cliniques par la disparition des trois lésions (Fig. 2). Une surveillance clinique, biologique et radiologique de plus de quatre ans ne montrera aucune récidive.

\section{Discussion}

Il s'agit donc d'un patient caucasien de 32 ans chez qui des nodules cérébraux sont mis en évidence dans l'hémisphère gauche, en frontal, pariétal et temporal, avec prise de contraste et effet de masse, au cours de l'exploration de symptômes neurologiques polymorphes. La recherche d'agents infectieux et l'analyse d'un fragment biopsié et d'une pièce d'exérèse $\mathrm{du}$ nodule frontal excluent toute origine infectieuse et néoplasique, mais plaide pour une atteinte démyélinisante de la substance blanche sans prolifération astrocytaire. En outre, des anticorps anti-VIH sont détectés chez ce patient dont l'immunité

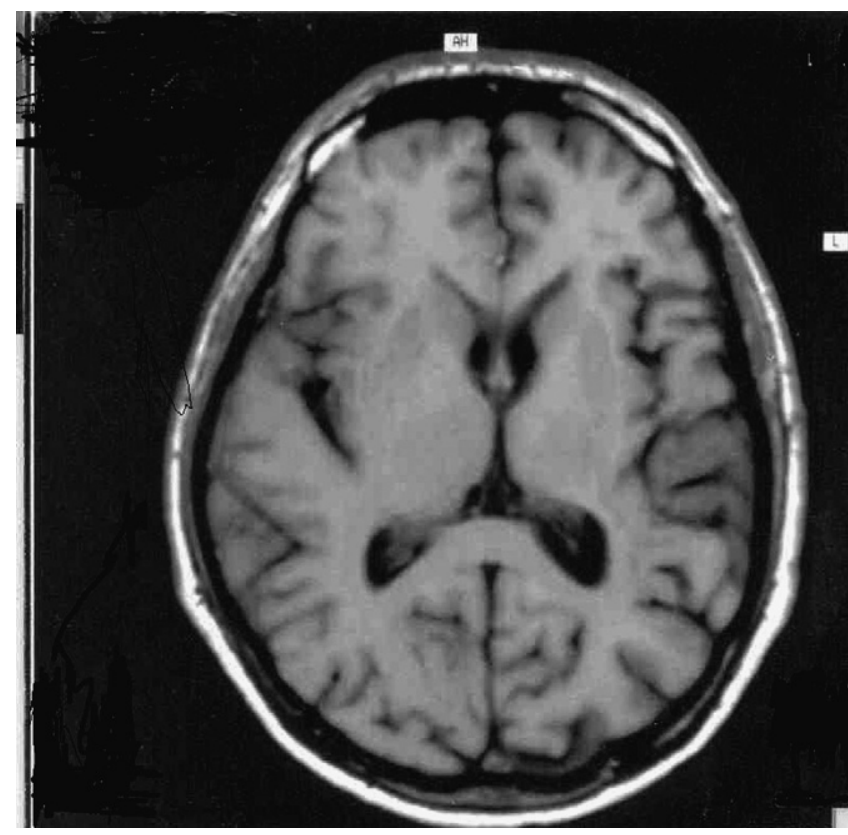

Fig. 2. Après exérèse partielle et traitement antirétroviral combiné, disparition des nodules précédemment observés.

After partial ablation and antiretroviral treatment, disappearance of the previously observed lesions.

est conservée. Après l'échec de la corticothérapie et de divers traitements anti- infectieux, la mise en route d'un traitement antirétroviral combiné va coïncider avec l'amélioration clinique et radiologique.

La description clinique du cas est intrigante : il y a une disparité entre les données cliniques, les résultats biologiques, les éléments radiologiques et histologiques et l'évolution sous traitement.

Primo, il convient d'interpréter l'atteinte démyélinisante de la substance blanche dans le contexte du VIH. Aussi, la LEMP paraît la pathologie la plus probable. En effet, comme nous l'avons déjà évoqué, elle produit une atteinte multifocale au niveau du carrefour ventriculaire, cependant sans effet de masse ni prise de contraste. Elle traduit souvent une immunodépression profonde et le virus JC est habituellement détecté. Exceptionnellement, elle a été décrite au cours de la primo-infection et en l'absence d'immunodépression. Chez notre patient, plusieurs éléments infirment la possibilité d'une LEMP, en particulier le siège des lésions radiologiques, leur prise de contraste et l'effet de masse qu'elles induisent.

Hormis la LEMP, l'atteinte cérébrale peut consister en une encéphalite $\mathrm{VIH}$ ou à des pathologies infectieuses de type toxoplasmose, cytomégalovirose, tuberculose ou tumorale, tel le lymphome cérébral primitif. Si les données cliniques et radiologiques corroborent une étiologie infectieuse ou tumorale, la biologie, l'histologie et l'évolution sous traitement ne plaident pas en faveur.

Les étiologies les plus communes étant réfutées, la recherche d'autres possibilités s'impose alors.

Ainsi, l'évocation d'une névrite optique en présence de symptômes neurologiques polymorphes nous oriente vers une atteinte 
démyélinisante du système nerveux central. La biologie ne contredit pas, de plus la nature des lésions à l'IRM, leur localisation et l'histologie permettent de conforter cette hypothèse. Dans ce cadre précis, trois affections peuvent être discutées: l'encéphalomyélite aiguë disséminée (EMAD) et sa variante évolutive multiphasique, l'encéphalomyélite aiguë nécrosante hémorragique et la SEP. Leur déterminisme postinfectieux et auto-immun constitue la caractéristique commune la plus évidente. L'EMAD nous semble l'entité la plus probable et peut donc être évoquée en premier lieu. Cette affection est caractérisée par une atteinte inflammatoire sévère de la substance blanche du cerveau ou de la moelle, d'origine auto-immune probable, habituellement secondaire à des maladies infectieuses aiguës virales ou à des vaccinations [6]. Théoriquement monophasique, l'EMAD peut cependant conduire à des récidives dont la traduction clinique et radiologique peut être similaire à celle présentée lors du premier épisode. Environ $30 \%$ des cas évoluent vers une SEP [7]. Il n'existe pas de marqueurs biologiques spécifiques; de ce fait, le diagnostic reste basé sur la clinique et la radiologie. Les récidives permettent de décrire une autre entité nosologique qui est l'encéphalomyélite multiphasique disséminée compatible avec notre patient. La description de notre cas retrouve en effet une évolution biphasique, proche aux plans cliniques, radiologiques et histologiques.

L'encéphalomyélite aiguë nécrosante hémorragique, qui est une forme plus agressive d'acute disseminated encephalomyelitis (EMAD), nous paraît très peu probable. Elle survient souvent après une infection des voies respiratoires supérieures. De début brutal, son évolution est foudroyante en une semaine, se manifestant par une destruction de la substance blanche du cerveau ou de la moelle, des foyers de nécrose et de hémorragies [6]. Ce tableau ne correspond pas aux éléments que nous avons rapportés de notre patient.

Enfin, il convient de s'attarder sur la SEP. Bien que la description histologique de notre patient ne soit pas typique d'une SEP, de fortes similitudes cliniques et radiologiques sont cependant notées. Aussi, il est permis d'évoquer une atteinte leucoencéphalitique mimant la SEP comme décrits chez de nombreux patients VIH-1 depuis une quinzaine d'années. Ainsi, diverses présentations de patients avec des symptômes de leucoencéphalopathie aiguë difficile à différentier de la SEP ont été faites. Ces symptômes apparaissaient soit concomitamment à la séroconversion VIH, soit endéans les trois mois [1-5,8,9]. Dans la majorité des cas, il existait une étroite relation temporelle entre le développement des symptômes neurologiques et la détermination de la séropositivité pour le VIH, indiquant, comme chez notre patient, un temps de latence très court entre l'acquisition du virus et la survenue des symptômes. Il demeure évident qu'une coïncidence entre les deux évènements ne peut être exclue.

D'une façon générale, ces affections démyélinisantes présentent des caractéristiques cliniques, radiologiques et histologiques quasi similaires à la présentation de notre patient. Divers traitements (corticoïdes, immunosuppresseurs, plasmaphérèse, interféron) vont produire des bénéfices variables sur ces affections. Chez notre patient, la régression de ces symptômes et la disparition des lésions cérébrales coïncident de façon étonnante avec la mise en route d'un traitement antirétroviral combiné. C'est bien la première fois qu'une telle analogie est constatée. L'implication du VIH dans la genèse et la perpétuation de la pathologie peut donc être évoquée, et ce, d'autant plus qu'aucun autre agent infectieux n'avait été retrouvé. Cependant, ce rôle n'est pas clair. L'étiologie infectieuse, en particulier virale, a souvent été suspectée lors d'atteintes démyélinisantes du SNC comme la SEP, l'EMAD, etc. Le VIH pourrait ainsi, dans notre cas, jouer un rôle par un mécanisme indirect plutôt que direct. Aucune particule virale n'ayant été retrouvée sur les pièces d'exérèse et l'absence d'immunodépression, c'est un mécanisme dysimmunitaire qui nous paraît le plus probable. Nous supposons que les effets délétères de la réplication virale sur le SNC vont être stoppés par le traitement combiné. Les lésions démyélinisantes constatées seraient donc la première manifestation de la séroconversion par un mécanisme auto-immun. L'inhibition de la réplication virale induite par le traitement combiné réduirait, voire annihilerait la virulence du VIH et, par la même, ses effets dysimmunitaires. De toute évidence, notre présentation constitue la première description de l'efficacité indirecte d'un traitement combiné sur des lésions cérébrales démyélinisantes chez un patient VIH+ immunocompétent.

\section{Références}

[1] Berger JR, Sheremata WA, Resnick L, Atherton S, Fletcher MA, Norenberg M. Multiple sclerosis-like illness occurring with human immunodeficiency virus infection. Neurology 1989;39:324-9.

[2] Gray F, Chimelli L, Mohr M, Clavelou P, Scaravilli F, Poirier J. Fulminating multiple sclerosis-like leucoencephalopathy revealing human immunodeficiency virus infection. Neurology 1991;41:105-9.

[3] Brew BJ. MS-like leukoencephalopathy in HIV-1. Neurology 1991;41: 1166.

[4] Graber P, Rosenmund A, Probst A, Zimmerli W. Multiple sclerosis-like illness in early HIV infection. AIDS 2000;14:2411-3.

[5] Facchini SA, Harding SA, Waldron RL. Human immunodeficiency virus1 infection and multiple sclerosis-like illness in a child. Pediatr Neurol 2002;26:231-5.

[6] Tenembaum S, Chitnis T, Ness J, Hahn JS, International Pediatric MS Study Group. Acute disseminated encephalomyelitis. Neurology 2007;68: 23-36.

[7] de Seze J, Debouverie M, Zephie H, et al. Acute fulminant demyelinating disease. A descriptive study of 60 patients. Arch Neurol 2007;64(10):1426-32.

[8] Duran E, Galvez J, Patrignani G, et al. Multiple sclerosis-like illness in a HIV-1 patient. J Neurol 2004;251:1142-4.

[9] Coban A, Akman-Demir G, Ozsut H, Eraksoy M. Multiple sclerosis-like clinical and magnetic resonance imaging findings in human immunodeficiency virus positive case. Neurologist 2007;13:154-7. 